\title{
Spindle cell lesions of the adult prostate
}

\author{
Donna E Hansel ${ }^{1}$, Mehsati Herawi ${ }^{1}$, Elizabeth Montgomery ${ }^{1}$ and Jonathan I Epstein ${ }^{1,2,3}$ \\ ${ }^{1}$ Department of Pathology, The Johns Hopkins Hospital, Baltimore, MD, USA; ${ }^{2}$ Department of Urology, \\ The Johns Hopkins Hospital, Baltimore, MD, USA and ${ }^{3}$ Department of Oncology, The Johns Hopkins \\ Hospital, Baltimore, MD, USA
}

\begin{abstract}
Prostatic spindle cell lesions are diagnostically challenging and encompass a broad array of benign and malignant processes. A subset of these lesions arises only within the prostate and generally represents entities that originate from the prostate epithelium or stroma, such as sclerosing adenosis, sarcomatoid carcinoma, stromal tumors of uncertain malignant potential (STUMP), and stromal sarcoma. Another subset of spindle cell tumors that involve the prostate are also found at other sites and include solitary fibrous tumor, leiomyosarcoma, and neural lesions among others. Finally, tumors may secondarily involve the prostate yet present as primary prostatic processes, as is evident with several cases of gastrointestinal stromal tumors (GIST). The utility of ancillary studies, including immunohistochemistry, is often limited and the main criteria for diagnosis are the morphologic findings by routine H\&E stain. This review addresses the various entities that may present as spindle cell tumors within the adult prostate and discusses the functional aspects of the differential diagnosis of these lesions.

Modern Pathology (2007) 20, 148-158. doi:10.1038/modpathol.3800676
\end{abstract}

Keywords: prostate; carcinoma; sarcoma; stroma; mesenchyme

Although relatively infrequent, spindle cell lesions of the prostate represent a diagnostically challenging and diverse array of entities. A subset of these lesions is specific to the prostate and includes stromal tumors of uncertain malignant potential (STUMP), stromal sarcoma and sarcomatoid carcinoma (carcinosarcoma) of the prostate. Additional lesions that may involve the prostate with some frequency, in addition to other anatomic sites, include inflammatory myofibroblastic tumor (IMT), solitary fibrous tumor (SFT), smooth muscle tumors, and rhabdomyosarcoma. Extension of a gastrointestinal stromal tumor (GIST) from the adjacent colon may rarely involve the prostate and even present clinically as an intraprostatic mass. Finally, sclerosing adenosis may occasionally mimic a spindle cell neoplasm. Morphologic assessment of the benign or malignant nature of the spindle cell component, the admixture of benign or malignant glands, and the presence of heterologous elements are all useful features in distinguishing these lesions from one another.

Correspondence: Dr JI Epstein, MD, Department of Pathology, The Johns Hopkins Hospital, 401 N. Broadway Street, Room 2242, Weinberg Building, Baltimore, MD 21231, USA.

E-mail: jepstein@jhmi.edu

Received 1 May 2006; revised 7 July 2006; accepted 25 July 2006
The majority of spindle cell lesions of the prostate are initially diagnosed on needle biopsy or transurethral resection (TUR) of the prostate, and the pathologist should be familiar with the broad differential diagnosis associated with these lesions. The diagnosis may be additionally challenging due to small quantities sampled by needle biopsy or cautery artifact associated with TUR specimens. Patient history is useful in certain instances, as many patients with sarcomatoid carcinoma have a prior documented history of prostatic acinar adenocarcinoma. However, the presence of an elevated serum prostate-specific antigen (PSA) level, abnormal digital rectal examination, or findings on imaging studies is nonspecific, and may be associated with many of these lesions. Patient age is generally not helpful, although the differential diagnosis for patients under the age of 20 years most commonly includes rhabdomyosarcoma, which is generally not seen in an older population, and occasionally inflammatory myofibroblastic tumor.

Owing to the rarity of these lesions, only a limited number of clinical or pathologic studies have been performed that address the morphologic spectrum and biologic behavior of these entities. This review is designed to present a comprehensive overview of the current literature concerning spindle cell lesions of the adult prostate, as well as provide a guide to their pathological differential diagnosis. 


\section{Spindle lesions unique to the prostate}

\section{Stromal Nodules of Hyperplasia}

The smallest nodules of benign prostatic hyperplasia $(\mathrm{BPH})$ are often stromal and are composed of loose mesenchymal tissue containing prominent small round vessels (Figures 1 and 2). Prostatic TURs can sometimes contain extensive stromal BPH that can be confused with a STUMP (see following section). The prominent vessels and multinodularity of stromal BPH distinguishes it from the myxoid pattern of STUMP that may consist of sheets of stroma. In a needle biopsy specimen, the prominent vessels of BPH help differentiate between a stromal nodule and a mesenchymal tumor. Stromal nodules of BPH may also contain an admixture of chronic inflammatory cells, which are typically lacking in STUMPs.

\section{STUMPs and Stromal Sarcomas}

Prostatic stromal tumors arising from the specialized prostatic stroma are rare and distinct tumors with diverse histological patterns. In the past, these tumors have been reported under a variety of terms including atypical stromal (smooth muscle) hyperplasia, phyllodes type of atypical stromal hyperplasia, phyllodes tumor, and cystic epithelial-stromal tumors. As the phyllodes 'leaf-like' pattern is only seen in a subset of both benign and malignant stromal tumors (see below), we prefer to designate stromal tumors of the prostates in more general descriptive terms as STUMPs and stromal sarcomas, as has also been recommended by the 2004 World Health Organization Classification of Tumours of the Urinary System and Male Genital Organs. ${ }^{1}$ To date, three studies have examined large patient populations in order to address the pathologic and clinical features associated with these lesions. ${ }^{2-4}$ Although STUMPs have been generally considered to variably represent either a hyperplastic or benign neoplastic stromal process, a subset of STUMPs has been associated with stromal sarcoma on concurrent biopsy material or has demonstrated stromal sarcoma on repeat biopsy, suggesting a malignant progression in at least some cases. ${ }^{2-4}$ In general, patients with a diagnosis of STUMP have a good prognosis, as most cases are confined to the prostate and rarely progress to sarcoma. In contrast, stromal sarcomas can extend out of the prostate and metastasize to distant sites, such as bone, lung, abdomen and retroperitoneum.

STUMPs have been reported to occur between the ages of 27 and 83 years, with a median age of 58 years and a peak incidence in the sixth and seventh decades. $^{2-4}$ Patients presented most commonly with urinary obstruction, followed by abnormal rectal examination, hematuria, hematospermia, rectal fullness, palpable rectal mass or elevated serum prostate specific antigen (PSA) levels. On gross examination, STUMPs appear white-tan and may demonstrate a solid or solid-cystic pattern with smooth-walled cysts filled with bloody, mucinous or clear fluid. These tumors may involve either the transition zone or the peripheral zone and may range in size from microscopic lesions (which are typically incidentally found) to large, cystic lesions up to $15 \mathrm{~cm}$ in size. ${ }^{4}$

Microscopically, four patterns of STUMP have been described and include (1) hypercellular stroma with scattered atypical, but degenerative appearing cells admixed with benign prostatic glands, (2) hypercellular stroma consisting of bland fusiform stromal cells admixed with benign glands, (3) leaflike hypocellular fibrous stroma covered by benign appearing prostatic epithelium similar in morphology to a benign phyllodes tumor of the breast, and (4) myxoid stroma containing bland stromal cells and often lacking admixed glands. Cases can exhibit a mixture of the above patterns. Mitotic activity is rare to absent in all of these subtypes and tumor necrosis is not present. Lesions may be present only focally on prostatic biopsies, and care must be taken to detect these lesions when evaluating prostate biopsies for other processes. There appears to be no correlation between the pattern of STUMP and association with stromal sarcoma.

Approximately half of all reported cases of STUMP demonstrate the first pattern of hypercellular stroma containing atypical cells intermixed with, but not compressing, benign glands (Figure 3). The atypical stromal cells in these cases are pleomorphic and hyperchromatic, with a marked degenerative appearance. Mitotic figures are typically absent and atypical mitoses should not be seen. Although the admixed glands resemble normal benign prostatic glands, the glands within a STUMP may appear more crowded than acini in the surrounding uninvolved prostate. The remaining three patterns of STUMP are seen with varying frequency. Cases of STUMP demonstrating hypercellular, elongated bland stromal cells with admixed glands may be occasionally misdiagnosed as a robust stromal proliferation associated with $\mathrm{BPH}$, although the extent of hypercellularity and often more eosinophilic nature of the cytoplasm is unique (Figure 4). The benign, phyllodes pattern of STUMP may also contain atypical, degenerative-appearing stromal cells and may be associated with a variety of benign epithelial proliferations, including basal cell hyperplasia, adenosis, and sclerosing adenosis (Figure 5). Finally, the myxoid pattern of STUMP may be confused with stromal nodules of $\mathrm{BPH}$, although the myxoid pattern of STUMP consists of extensive sheets of myxoid stroma without the nodularity identified in BPH or occasionally myxoid stroma admixed with benign prostate glands (Figure 6).

Immunohistochemical stains do not necessarily aid in distinguishing STUMPs from other spindle cell lesions of the prostate. Most cases of STUMP are 


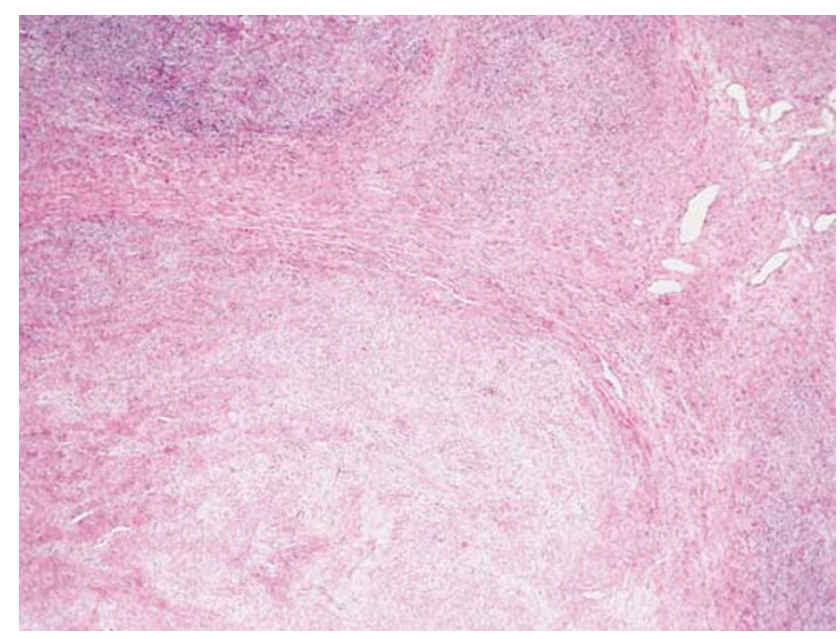

Figure 1 Low magnification of a stromal nodule of $\mathrm{BPH}$ demonstrating prominent nodularity.

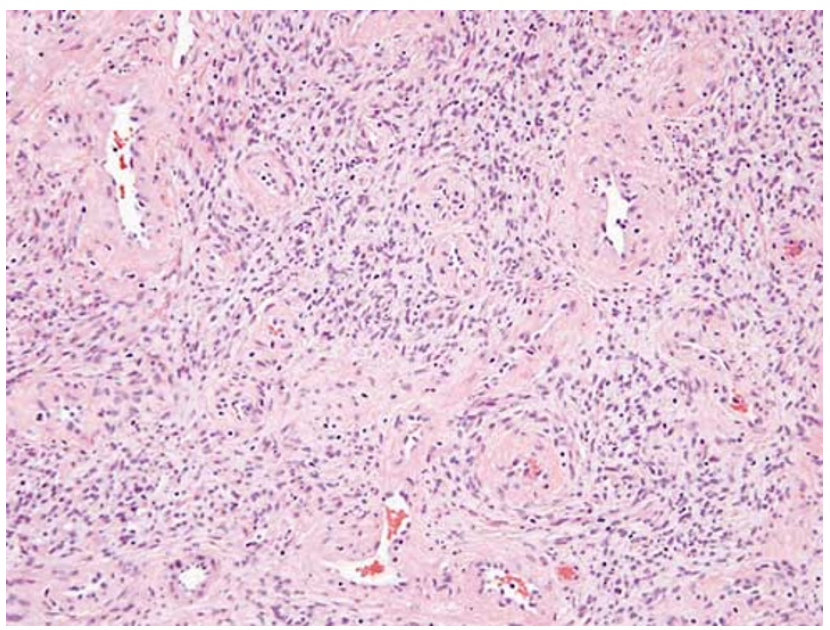

Figure 2 High magnification of a stromal nodule of BPH showing bland spindled cells and prominent vascularity.

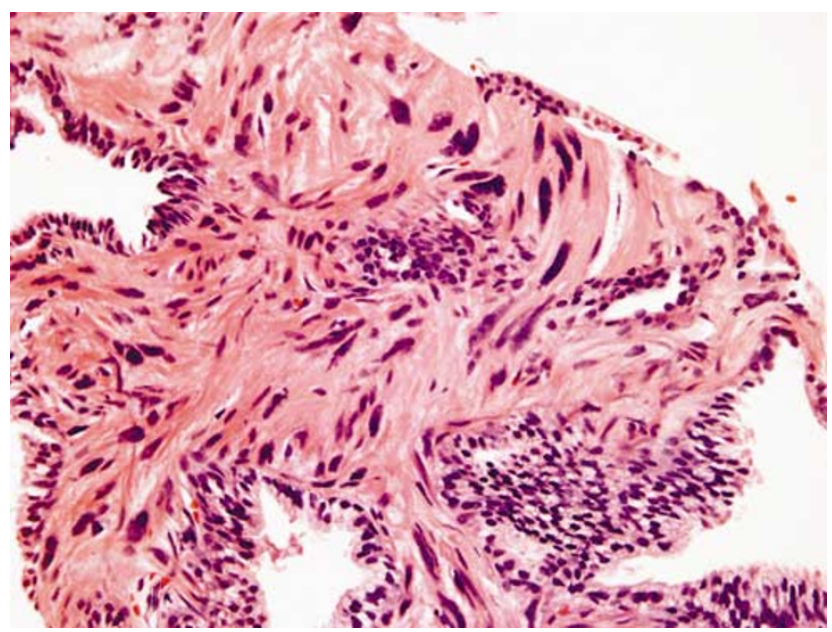

Figure 3 STUMP with enlarged, degenerative-appearing cells interspersed among benign prostatic glands.

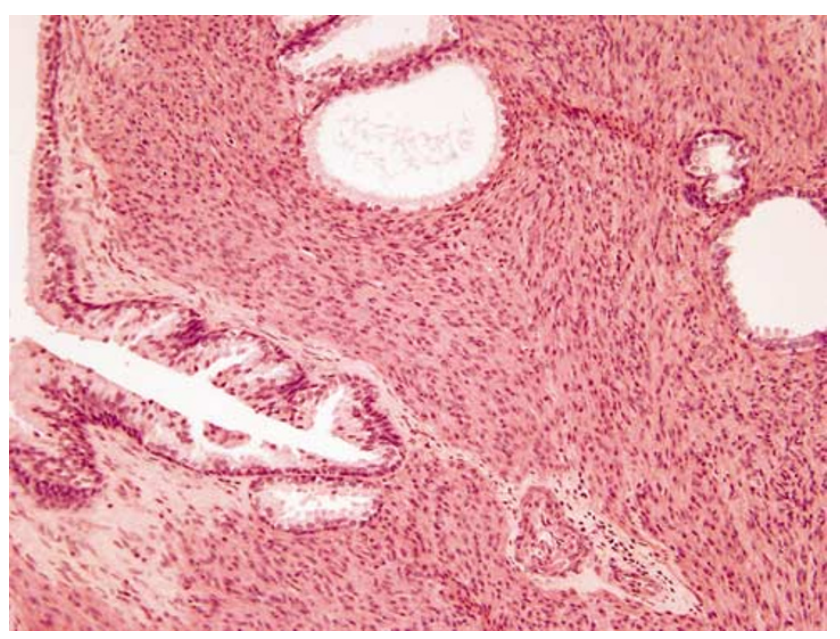

Figure 4 Hypercellular STUMP surrounding benign prostatic glands.

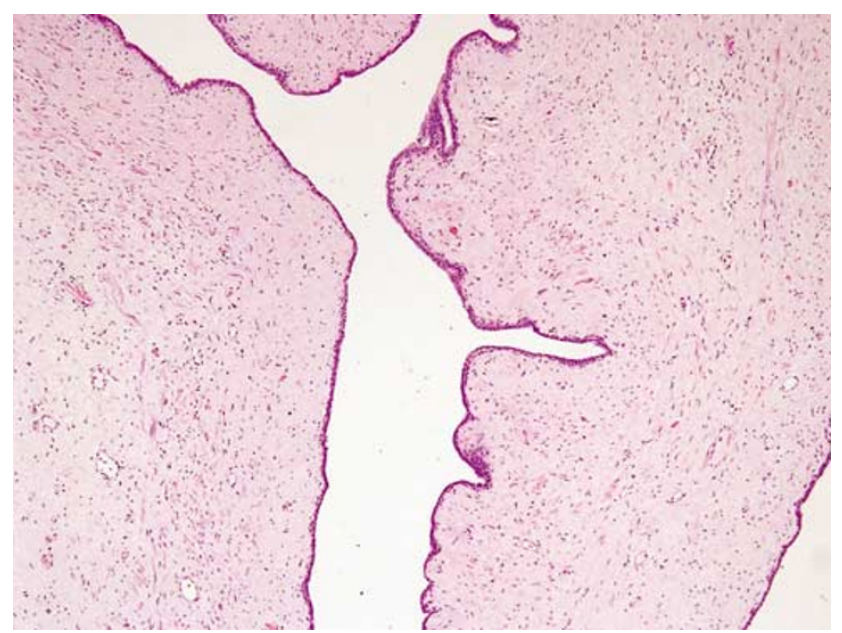

Figure 5 Benign phyllodes-like STUMP with overlying lining of benign prostatic epithelium.

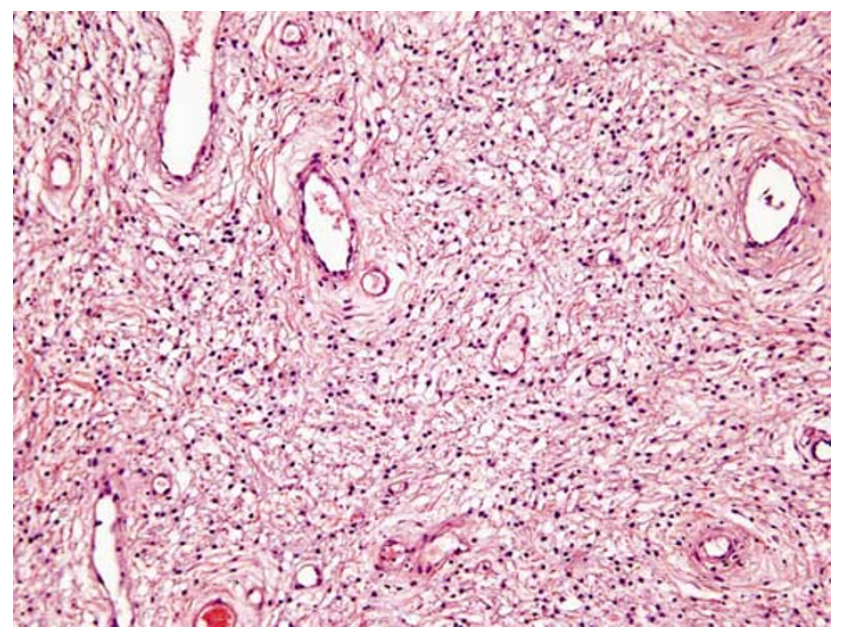

Figure 6 Myxoid STUMP showing bland spindled cells in a background of myxoid matrix and vasculature. 
positive for CD34 and vimentin and variably positive for smooth muscle actin, desmin, and HHF-35. Owing to the derivation of these tumors from the prostatic stroma, ${ }^{5-7}$ progesterone receptor is frequently present on immunostaining, although estrogen receptor is less commonly positive. C-kit and S-100 were negative in all cases examined.

In contrast to STUMPs, stromal sarcomas tend to affect a slightly younger population, with a reported age range of 25-86 years. Approximately half of all reported cases of stromal sarcoma occur before the age of 50 years. Stromal sarcomas may arise de novo or may exist in association with either a pre-existent or concurrent STUMP, thereby suggesting a potential for STUMPs to dedifferentiate to sarcomas in rare cases.

Gross examination of stromal sarcomas demonstrates predominantly tan-white, solid, fleshy lesions ranging in size from 2 to $18 \mathrm{~cm}$. Occasionally, areas of edema, hemorrhage, or small cysts may be identified. Microscopically, stromal sarcomas demonstrate either a solid growth of neoplastic stromal cells, which may have storiform, epithelioid, fibrosarcomatous, or patternless patterns, or may intercalate between benign prostatic glands (Figure 7). Less commonly stromal sarcomas may demonstrate leaf-like glands with underlying hypercellular stroma, which are also termed malignant phyllodes tumors (Figure 8). Stromal sarcomas have one or more of the following features within the spindle cell component: hypercellularity, cytological atypia, mitotic figures, and necrosis. Stromal sarcomas may additionally be subclassified into low and high grades with high-grade tumors defined by moderatemarked pleomorphism and hypercellularity often with increased mitotic activity and occasional necrosis.

Immunohistochemical findings are similar to those of STUMPs, with strong vimentin reactivity and positivity for CD34 and progesterone receptor. In a subset of cases studied, pancytokeratin and CAM5.2 was negative. One case of stromal sarcoma was reported to demonstrate nuclear reactivity for beta-catenin, although the significance of this finding is unclear.

The variability in behavior of STUMPs and stromal sarcomas, and their occasional co-existence, lead to challenges in patient management. Lowgrade sarcoma can locally invade, despite having at times relatively bland cytology, and high-grade sarcoma has the potential to metastasize to distant sites. Although many STUMPs may behave in an indolent fashion, their unpredictability in a minority of cases and the lack of correlation between different histological patterns of STUMPs and sarcomatous dedifferentiation, warrant close follow-up and consideration of definitive resection in younger individuals. Factors to consider in deciding whether to proceed with definitive resection for STUMPs diagnosed on biopsy include patient age and treatment preference, presence and size of the lesion on rectal exam or imaging studies, and extent of the lesion on tissue sampling. Expectant management with close clinical follow-up could be considered in an older individual with a limited lesion on biopsy where there is no lesion identified on digital rectal exam or on imaging studies.

\section{Sarcomatoid Carcinoma of the Prostate}

Sarcomatoid carcinoma (carcinosarcoma) is a rare type of prostatic cancer with only approximately 100 cases reported in the literature and three large case series consisting of 42, 21, and 12 patients, respectively. ${ }^{8-10}$ Tumors are most commonly composed of an admixture of both malignant glandular and spindle cell elements, in which cases with predominantly a sarcomatoid component may be mistaken for a sarcoma. In support of the term 'sarcomatoid carcinoma' it has been demonstrated that both the malignant epithelial and spindle cell components are clonally related. ${ }^{11}$

Patients with sarcomatoid carcinoma often have a history of acinar adenocarcinoma of the prostate, although in some cases the diagnosis may have been as remote as 16 years prior. In one study that examined a large number of patients with sarcomatoid carcinoma, the vast majority of patients with known treatment history following the original diagnosis of acinar adenocarcinoma had received external beam radiation, hormonal therapy, a combination of external beam radiation and hormone therapy, or brachytherapy. ${ }^{8}$ The time between the original diagnosis of acinar adenocarcinoma and diagnosis of sarcomatoid carcinoma ranged from 6 months to 16 years (mean 6.8 years). The mean age of presentation was 70 years. Patients with sarcomatoid carcinoma have poor outcomes, with an actuarial risk of death of $20 \%$ within 1 year of diagnosis and frequent widespread metastases to sites including bone, liver, and lung. ${ }^{8}$

Morphologically, sarcomatoid carcinoma demonstrates a variety of patterns. Typically, the glandular component is composed of high-grade acinar adenocarcinoma or an unusual subtype of prostatic carcinoma, including small cell, foamy gland, basaloid, ductal, squamous, and adenosquamous carcinoma, among others (Figure 9). The sarcomatoid component can vary from 5 to $99 \%$ and demonstrates malignant features including hypercellularity, enlarged hyperchromatic nuclei, frequent mitoses and occasional necrosis. Bizarre tumor giant cells may be present within the sarcomatoid component. In approximately $10 \%$ of sarcomatoid carcinomas, the sarcomatoid component has at most mild atypia. ${ }^{8}$ In approximately $30 \%$ of cases, heterologous elements of osteosarcoma, chondrosarcoma, or rhabdomyosarcoma are also present, which may aid in the diagnosis. The rationale for considering carcinosarcoma and sarcomatoid carcinoma as one entity is that the prognosis 
152

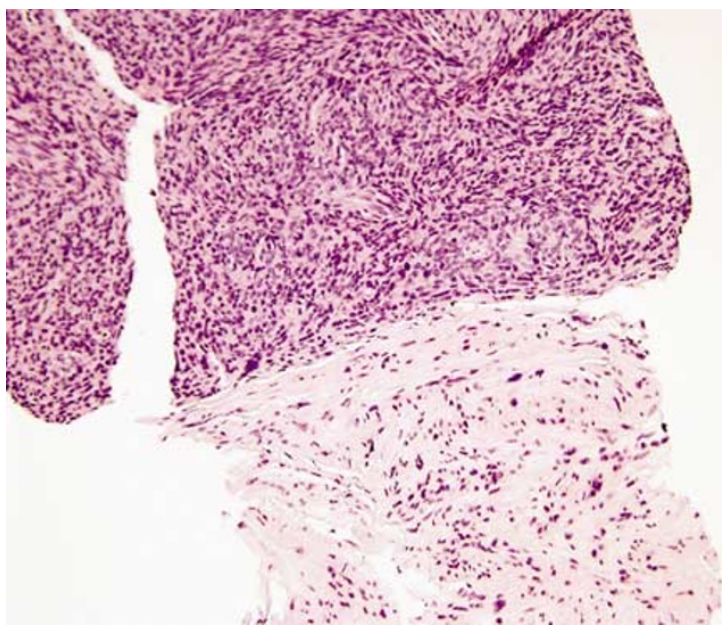

Figure 7 Stromal sarcoma (top) adjacent to degenerative-type STUMP (bottom).

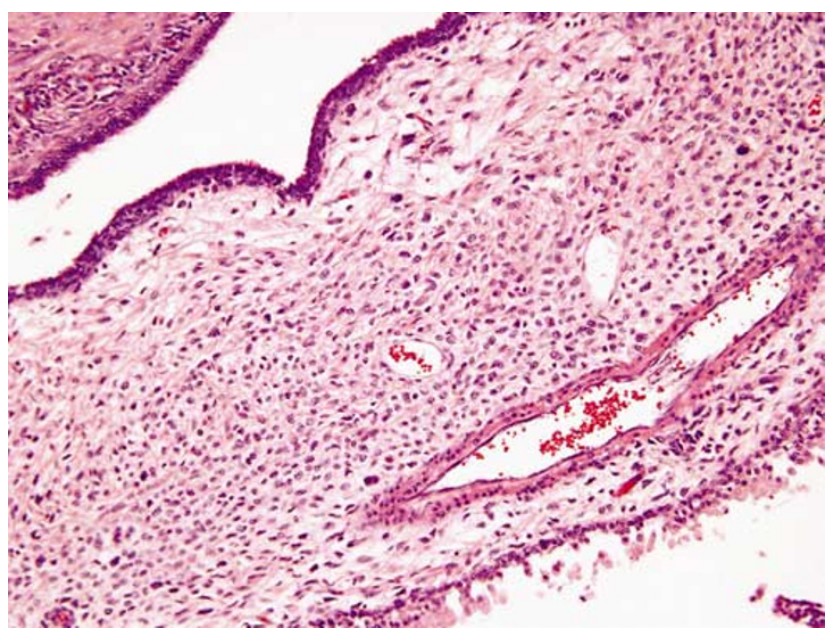

Figure 8 Malignant phyllodes-pattern stromal sarcoma demonstrating hypercellular, atypical stromal cells with increased mitotic activity. The leaf-like elements are lined by benign prostatic epithelium.

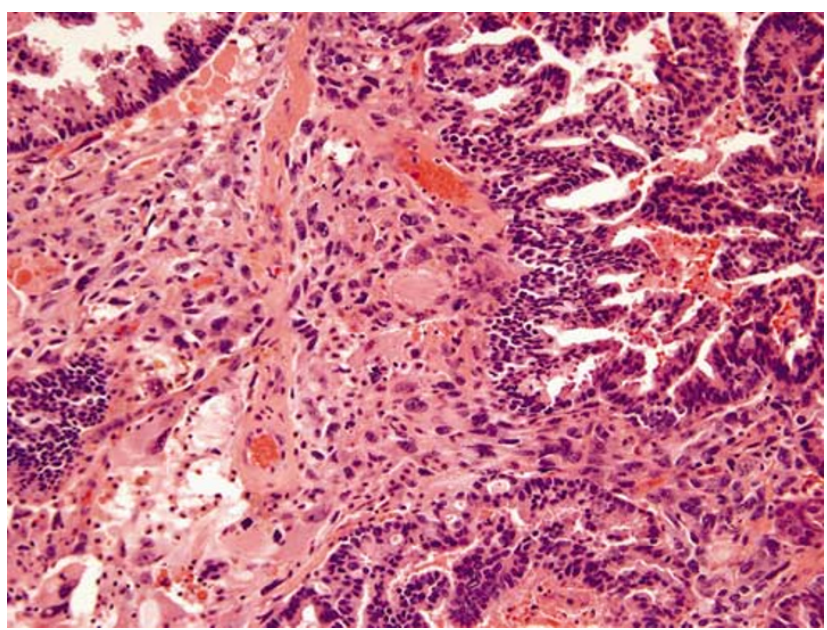

Figure 9 Sarcomatoid carcinoma (carcinosarcoma) containing an admixture of ductal adenocarcinoma of the prostate and a malignant spindle cell component. is the same regardless of whether heterologous elements are present. Also, even in cases with heterologous elements, the spindle cell component often expresses keratin suggesting a common origin rather than a collision tumor composed of sarcoma and carcinoma.

In some cases, there is a recent ( $<2$ years) history of adenocarcinoma of the prostate treated by radiation with recurrent tumor composed of a pure spindle cell population. The tumor may not fit into any of the typical patterns of sarcomas that are seen in the prostate (ie lacks long intersecting fascicles of fusiform cells of leiomyosarcoma) or may show heterologous differentiation (ie osteosarcoma). Even if these lesions do not express keratins, the likelihood is that they are sarcomatoid carcinomas rather than true sarcomas. Post-radiation sarcomas typically occur several years after treatment and it is recognized that many overt sarcomatoid sarcomas only focally express keratin in the spindle cell component.

\section{Sclerosing Adenosis}

Sclerosing adenosis is a benign prostatic proliferation of glands and stroma that frequently mimics acinar adenocarcinoma, but which may also mimic sarcomatoid carcinoma. Sclerosing adenosis is found incidentally most commonly in approximately $2 \%$ of TUR or prostatectomy specimens as single or multiple foci of well-formed small glands, scattered single cells, and a cellular spindle cell component. It is only rarely seen on needle biopsy. ${ }^{12-14}$ The glandular element, which is virtually always present, demonstrates crowding and an infiltrative pattern, and can show nuclear atypia consisting of nuclear enlargement with prominent nucleoli. Stains for basal cells are positive in the glandular component in sclerosing adenosis, in contrast to absent staining in cancerous glands in sarcomatoid carcinoma. Also, sclerosing adenosis tends to be a more focal lesion and the spindle cell component lacks atypia as compared to sarcomatoid carcinoma. The spindle cell element associated with these lesions is thought to be derived from myoepithelial cells surrounding the prostatic glands, as stains for high molecular weight cytokeratin and muscle-specific actin are often positive.

\section{Spindle lesions not unique to the prostate}

\section{Leiomyoma/Leiomyosarcoma}

The differential diagnosis of a leiomyoma $v s$ a stromal nodule in benign hyperplasia may be difficult, as small leiomyomas may be incidental findings on prostatic sampling that morphologically mimic a stromal nodule. Both leiomyomas and stromal nodules may contain abundant smooth muscle, although leiomyomas typically demonstrate well-organized fascicles that are not commonly seen in stromal nodules. Large single leiomyomas that are 
symptomatic are rare. Leiomyomas demonstrate virtually no mitotic activity and minimal to no nuclear atypia, with the exception of occasional scattered degenerative nuclei in a normocellular background (Figure 10). This contrasts with the findings in leiomyosarcoma, which demonstrates varying degrees of atypia, mitotic activity, hypercellularity and necrosis.

Leiomyosarcoma of the prostate is rare, affecting men between the ages of 40-78 years and most frequently presents with urinary obstruction, as well as perineal/pelvic pain, urinary frequency, hematuria, constipation, rectal pain, and pain or burning on ejaculation. ${ }^{15,16}$ Lesions may range in size from 3 to $21 \mathrm{~cm}$ and are often highly infiltrative. Microscopically, these hypercellular lesions are composed of intersecting bundles of spindled cells with atypia ranging from moderate to severe (Figure 11). The vast majority of leiomyosarcomas in the literature have been high grade with frequent mitoses and necrosis, although we have also seen rare cases of low-grade prostatic leiomyosarcoma. Low-grade leiomyosarcomas are distinguished from leiomyomas by moderate amount of atypia, focal areas of increased cellularity, scattered mitotic figures, and a focally infiltrative growth pattern around benign prostate glands at the perimeter. Symplastic leiomyomas have, in contrast, scattered atypia of a degenerative nature with an overall low cellularity. As opposed to some stromal sarcomas, leiomyosarcomas lack admixed normal glands, except entrapped glands at the periphery. Similarly, sarcomatoid carcinomas differ from leiomyosarcomas in that they often contain admixed malignant prostatic glands.

Leiomyosarcomas commonly express vimentin, actin, and desmin. Cytokeratin expression is observed in about one-quarter of cases. ${ }^{17}$ In addition, some leiomyosarcomas have been reported to express the progesterone receptor, similar to STUMPs and stromal sarcomas. ${ }^{18}$ Patients with leiomyosarcoma commonly have a poor outcome, with 50$75 \%$ of patients dying from disease within $2-5$ years. The remaining patients frequently develop metastatic spread of disease to the lungs, often several years following initial diagnosis. In the study by Sexton et $a l,{ }^{16}$ the prognosis for leiomyosarcoma of the prostate, as for sarcomas of the prostate in general, was not dependent on stage, with the exception of a better prognosis for those men who presented without distant metastases. The only other variable that these authors found to be predictive of a favorable prognosis was complete surgical resection with microscopically negative margins. Optimal treatment requires a multimodal approach rather than surgery alone.

\section{Rhabdomyosarcoma}

The vast majority of rhabdomyosarcomas of the prostate occur in the pediatric population. ${ }^{19}$ There are $<20$ prostatic rhabdomyosarcomas that have been reported in adults ranging in age from 17 to 68 years old..$^{20,21}$ As in younger patients, embryonal subtype is the predominant pattern. In contrast to the relatively good prognosis for treated rhabdomyosarcoma in children, the prognosis in adults is poor with most dying of disease in $<2$ years, despite multimodality therapy. In part, the dismal prognosis is due to delayed presentation and extensive spread of disease at presentation. In tumors that are better differentiated, recognition of rhabdomyoblasts directs the pathologist to the correct diagnosis. In more primitive tumors, immunohistochemistry for skeletal muscle specific markers, such as myogenin or myo-D1 can help to identify the tumor as rhabdomyosarcoma.

\section{Inflammatory Myofibroblastic Tumor}

Inflammatory myofibroblastic tumor (IMT) is described by a variety of terms in the literature, including pseudosarcomatous fibromyxoid tumor and 'post-operative' spindle cell nodule. ${ }^{22-24}$ Although lesions occurring in the setting of prior instrumentation of the prostate and those arising as de novo prostatic lesions have in the past been considered as separate entities, it is now considered that both are IMTs. Regardless of whether there is or there is not a prior history of instrumentation, lesions have overlapping morphological, immunohistochemical, and molecular features and demonstrate the same clinical behavior. IMTs of the prostate have been reported in men ranging in age from 42 to 67 years, ${ }^{24,25}$ although IMTs within the bladder have been reported in patients between 3 and 86 years of age. ${ }^{24}$ In some cases, it is difficult to distinguish whether lesions arise in the bladder or prostate.

In contrast to many other spindle cell lesions of the prostate, IMTs may be fairly small, with many cases $<1 \mathrm{~cm}$ in size. IMTs of the prostate are, for the most part, morphologically identical to those found at other sites and show uniform, but reactive, myofibroblasts with a cell-culture appearance in a background of variable inflammation, prominent vascularity, and extravasated red blood cells (Figure 12). Mitotic rate is variable but may be elevated; abnormal mitoses are not seen.

IMTs commonly express ALK, pancytokeratin (81\%), Cam5.2 (56\%), actin (100\%), desmin (80\%), and p53 (93\%) by immunohistochemistry. ${ }^{26}$ ALK gene fusion at chromosome 2p23 is identified by fluorescence in situ hybridization (FISH) in approximately $75 \%$ of IMTs of the genitourinary tract and often correlates with protein expression by immunohistochemistry. ${ }^{24,26}$ IMTs are generally negative for S100, CD34, CD117, CD21, and CD23. Most IMTs follow a benign course, although incomplete surgical resection may lead to recurrence in approximately one quarter of cases. Lesions may extend outside of the prostate. With the exception of the following unique case, IMTs have not been reported 


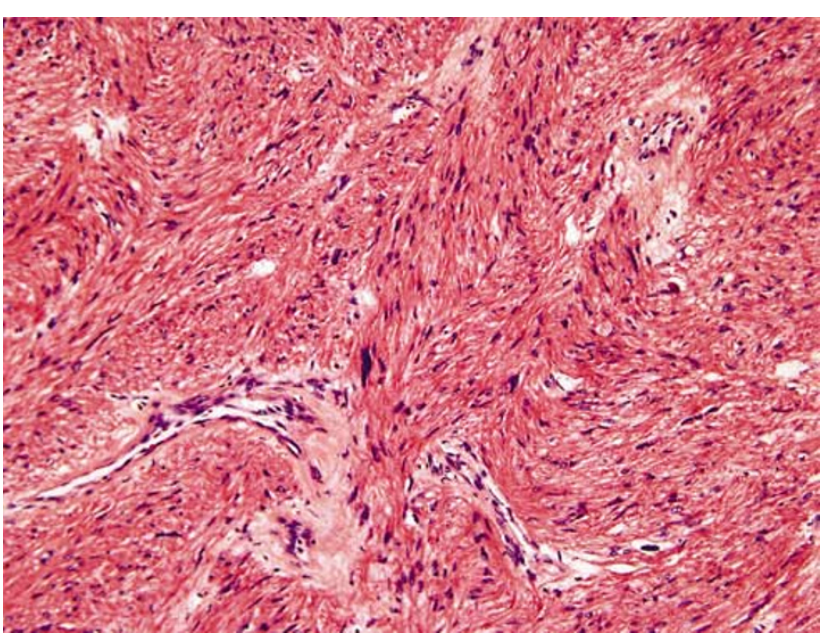

Figure 10 Symplastic leiomyoma demonstrating a fascicular pattern with occasional cells with degenerative-appearing atypia.

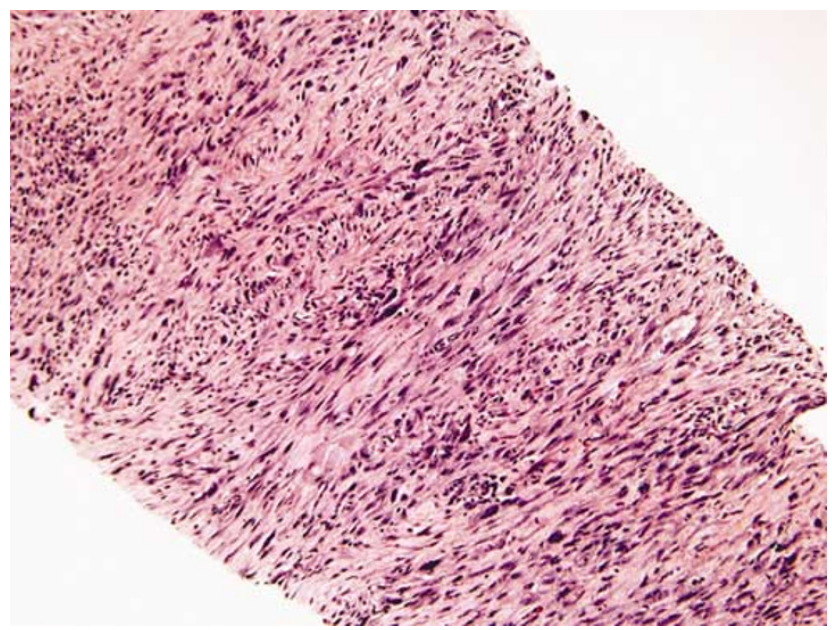

Figure 11 Needle biopsy of a high-grade leiomyosarcoma showing the characteristic fascicular pattern with marked nuclear atypia and hypercellularity.

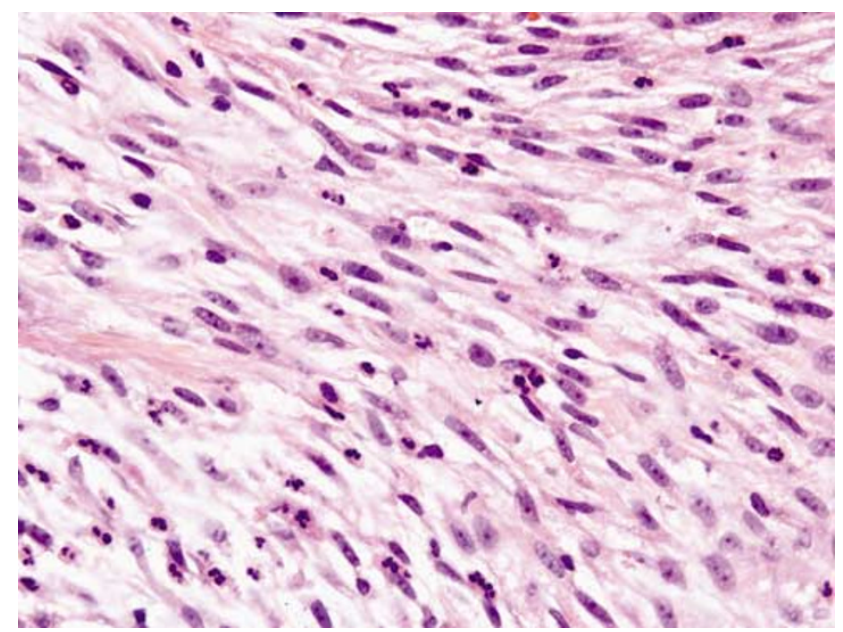

Figure 12 High-magnification view of an IMT with cells containing tissue-culture-like myofibroblasts with uniform nuclei lacking hyperchromatism admixed with inflammatory cells and occasional extravasated red blood cells. to metastasize. We have seen a case involving the prostatic urethra with the overall morphology of IMT and having an ALK gene rearrangement, yet had malignant features with atypical mitotic figures and hypercellularity. The tumor recurred following cystoprostatectomy and the patient subsequently developed intra-abdominal metastases and died 9 months following surgery.

\section{Solitary Fibrous Tumor}

Solitary fibrous tumor (SFT) occurs most commonly along the pleura, but may occur in a variety of extrathoracic sites including the genitourinary tract. There are fewer than 20 cases of SFT involving the prostate reported as single cases and one series of 12 cases. ${ }^{27-34}$ Prostatic SFTs have been reported in patients ranging in age from 21 to 75 years and the most common clinical findings included urinary retention, urinary frequency, dysuria, constipation, incontinence and groin pain.

These tumors demonstrate a broad size distribution, ranging from 2 to $14 \mathrm{~cm}$, with many reported to be $>5 \mathrm{~cm}$. Microscopically, prostatic SFTs appear similar to those identified in extraprostatic sites. Uniform spindled cells with bland nuclei are arranged in a 'patternless' pattern in a background of variable ropy collagen (Figure 13). Many cases demonstrate a hemangiopericytomatous appearance. Admixed prostatic tissue is not commonly associated with these lesions. Malignant behavior may be heralded by the findings of hypercellularity, pleomorphism, necrosis and infiltrative margins.

Immunohistochemistry generally reveals diffuse reactivity for CD34, vimentin and bcl-2, although rare SFTs may lack some of these markers. Staining for CD99, beta-catenin, p53, smooth muscle actin, and muscle-specific actin has also been reported. These tumors are typically negative for pancytokeratin, S-100, and CD117 (c-kit).

\section{Gastrointestinal Stromal Tumor}

Although gastrointestinal stromal tumors (GISTs) lesions may clinically present as primary prostatic processes on imaging studies and clinical exam, such cases are typically large masses arising from the rectum or perirectal space that compress but do not invade the prostate. Exceptionally, they may also invade the prostate. ${ }^{35-37}$ Most cases of 'prostatic' GISTs are sampled on needle biopsy, although we have seen one case sampled on a transurethral resection. Typically GIST is not considered in the differential diagnosis of spindle cell lesions of the prostate, although the unique management of these tumors underscores the importance of recognizing these tumors. Misdiagnosis of GISTs involving the prostate has been reported and we have seen similar cases, and several patients have undergone pelvic 
exenteration, irradiation, and chemotherapy for a misdiagnosis of pelvic sarcoma. ${ }^{36}$

Two case reports and a series of eight cases have described GISTs established on prostatic biopsy or TUR specimens. ${ }^{35-37}$ Patients ranged in age from 42 to 65 years and presented with urinary obstructive symptoms, rectal fullness, and abnormal digital rectal examination. Tumor size ranged from 1 to $14 \mathrm{~cm}$. Microscopically, 'prostatic' GISTs are morphologically identical to those found within the gastrointestinal tract. Spindled cells with a fascicular growth pattern, perinuclear vacuoles, occasional focal epithelioid features and variable necrosis were identified (Figure 14). Three of eight cases reported in one series had malignant features.

CD117/c-kit was uniformly expressed in all cases and CD34 was positive in seven of eight cases studied. S100, desmin and smooth muscle actin were negative. On prostate biopsy, it may be difficult to distinguish a GIST from other spindle cell

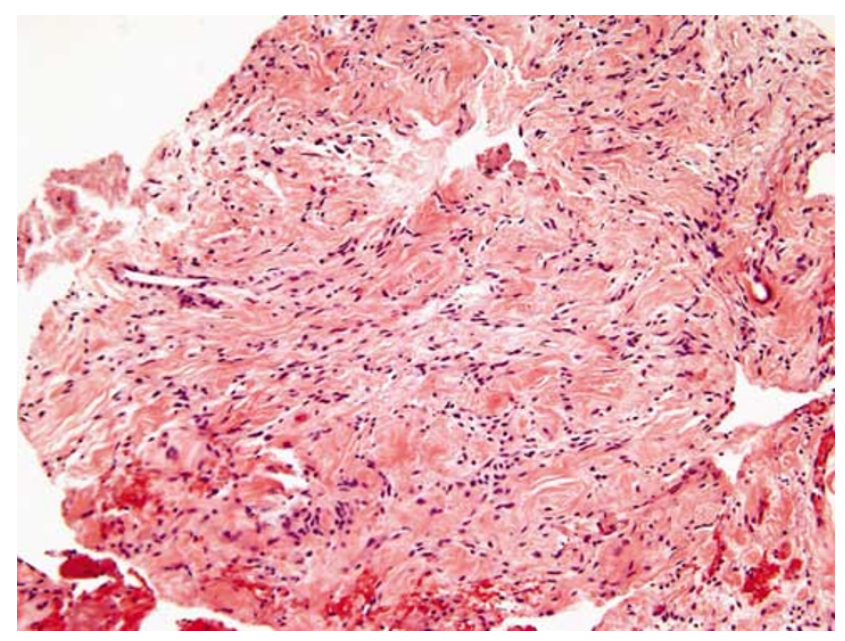

Figure 13 Solitary fibrous tumor on needle biopsy showing small, uniform cells intermingled with thick, 'ropy' collagen.

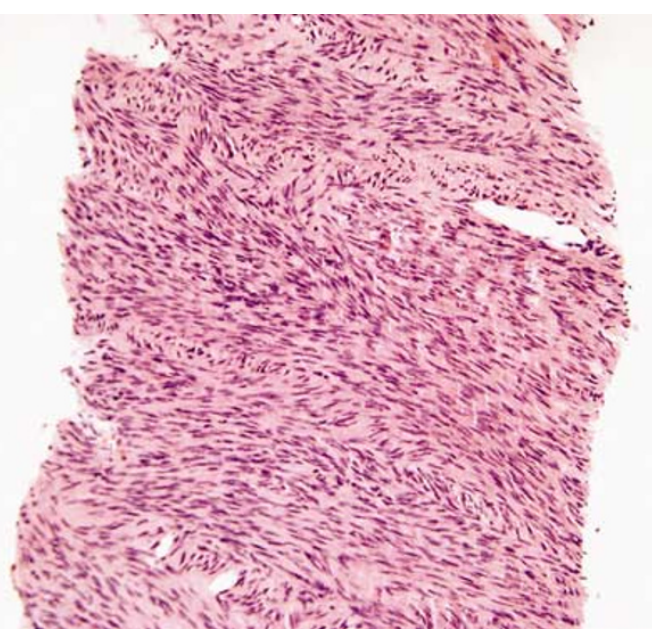

Figure 14 GIST identified on needle biopsy. tumors due to limited material. Consequently, prior to rendering a diagnosis of SFT, schwannoma, leiomyosarcoma, or stromal sarcoma, GIST should be considered in the differential diagnosis. Furthermore, immunostains for CD117 should be performed to verify the diagnosis. A subset of patients treated with the c-kit tyrosine kinase inhibitor imatinib (Gleevec) following the diagnosis of 'prostatic' GIST have demonstrated a subsequent reduction in tumor size ${ }^{37}$ No long-term follow-up is currently available on these patients to determine if the biologic behavior of GISTs secondarily involving the prostate is different than that described in other sites.

\section{Miscellaneous Lesions}

Other sarcomas involving the prostate that have been described as case reports include synovial sarcoma,${ }^{38-40}$ osteogenic sarcoma, ${ }^{41}$ malignant fibrous histiocytoma,${ }^{42-44}$ angiosarcoma ${ }^{45}$ neurofibroma, ${ }^{46}$

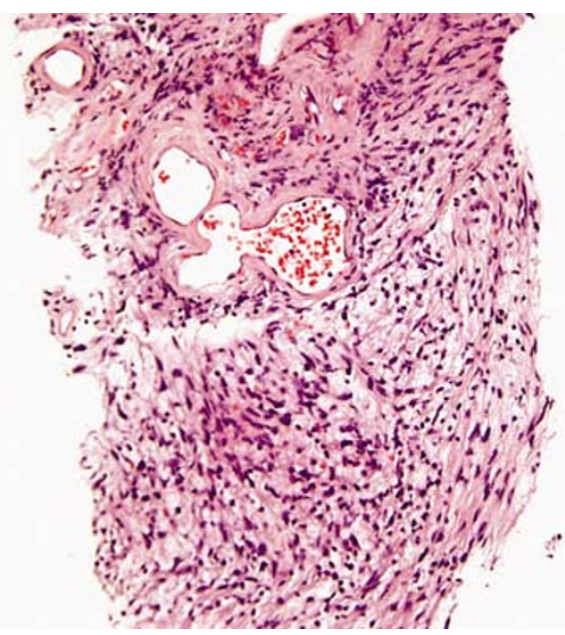

Figure 15 Cellular schwannoma diagnosed on needle biopsy.

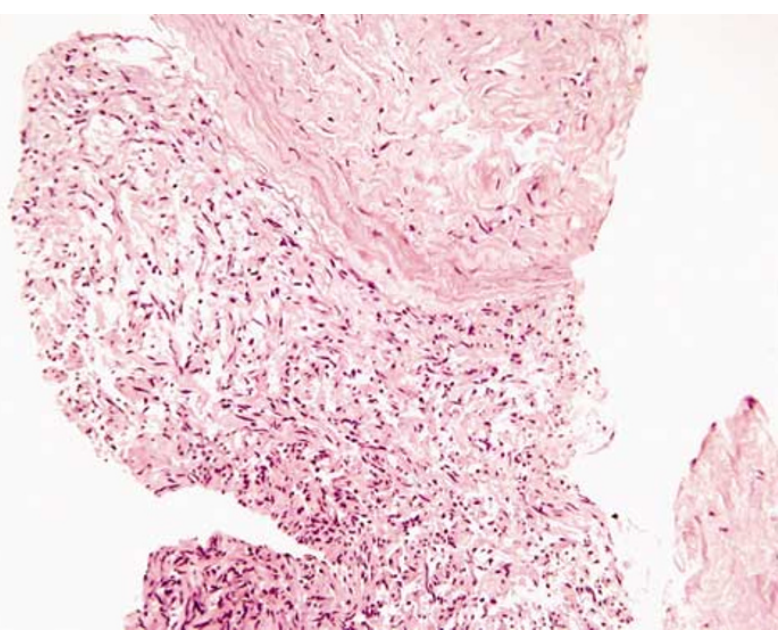

Figure 16 Neurofibroma demonstrating spindled endoneurial cells with poorly defined cell borders in a background of collagen. 
and malignant peripheral nerve sheath tumors. ${ }^{47}$ A single case of granular cell tumor of the prostate has also been recorded. ${ }^{48}$

We have seen in our practice (unpublished results) four nerve sheath tumors. One schwannoma was a small incidental lesion arising from the nerves adjacent to the prostate at radical prostatectomy. The other two schwannomas were seen on prostate needle biopsy, one was classic and the other cellular (Figure 15). The cellular schwannoma displayed strong S100 expression and no immunoreactivity for CD34. A neurofibroma was also seen on prostate needle biopsy and was S100 positive (Figure 16).

\section{Differential diagnosis and utility of ancillary studies}

The differential diagnosis of spindle cell lesions of the prostate remains challenging. In most cases, patients are middle-aged to elderly men, who present with nonspecific symptoms such as urinary tract obstruction, hematuria, and rectal fullness. Many patients have an abnormal digital rectal exam and serum PSA levels may be elevated. Imaging, when performed, lacks specificity both for the type of lesion and its malignant potential. Furthermore, imaging may not reliably distinguish the site of origin of the lesion. Occasionally, a history of prostate cancer may aid in the diagnosis of sarcomatoid carcinoma of the prostate, although the history may be unavailable or remote.
Grossly, lesional size may vary tremendously, irrespective of the type of lesion and whether it is benign or malignant. Infiltrative borders are also not specific for malignancy as some prostatic sarcomas can have a circumscribed growth pattern and lesions with a typically benign course, such as IMT, may appear infiltrative.

The most important tools in the differential diagnosis of spindle cell lesions of the prostate are the morphologic features and an awareness of the various entities that may occur in this location (Table 1). The presence of admixed prostate glands within the spindle cell lesion raises a more specific differential diagnosis, including some patterns of STUMP, some stromal sarcomas (malignant phyllodes pattern), sarcomatoid carcinoma and sclerosing adenosis. In sclerosing adenosis, STUMPs and stromal sarcoma, the glandular component is typically benign, whereas sarcomatoid carcinoma demonstrates admixed adenocarcinoma. In contrast, many of the remaining lesions, including IMTs, leiomyosarcomas, GISTs and SFTs generally grow in an expansile pattern, without admixed glandular elements. In these pure spindle cell lesions, individual cytological features and growth pattern, as described above, are important in correctly identifying the entity.

Immunohistochemistry occasionally aids in the diagnosis of spindle cell lesions (Table 2). GISTs are strongly positive for CD117/c-kit. Approximately $75 \%$ of IMTs demonstrate ALK-1 immunoreactivity or gene alterations by FISH. The majority of other immunohistochemical markers are variably present in many of the tumors, including CD34, actin, and progesterone receptor.

Table 1 Morphologic comparison of spindle lesions of the prostate

Morphology

STUMP

Stromal sarcoma

Sarcomatoid carcinoma

Sclerosing adenosis

Leiomyoma

Leiomyosarcoma

Rhabdomyosarcoma

IMT

Solitary fibrous tumor
Four patterns include (1) degenerative atypia, (2) hypercellular stroma, (3) benign phyllodes-like, (4) myxoid stroma with bland cells

Two patterns include (1) solid growth of overtly malignant cells with storiform, epithelioid, fibrosarcomatous, or patternless patterns, (2) malignant phyllodes-like

Admixture of high grade prostatic adenocarcinoma and spindled 'sarcomatoid' component with variable heterologous element formation

Foci of well-formed glands and single cells with cellular, spindled stroma

Well-organized smooth muscle fascicles lacking mitotic activity and with little to no atypia

Intersecting fascicles showing mitoses, cytologic atypia and necrosis

Primarily embryonal subtype consisting of small round cells with variable eosinophilic cytoplasm, occasional elongated/strap cells and, commonly, a myxoid background

Uniform, reactive myofibroblasts with a cell-culture appearance in a background of variable inflammation and extravasated red blood cells; often ALK positive

Uniform spindled cells with bland nuclei in a 'patternless' pattern among a background of ropy collagen

GIST 
Table 2 Immunohistochemical characteristics of nonepithelial prostatic spindle cell lesions

\begin{tabular}{|c|c|c|c|c|c|c|c|}
\hline & STUMP & $S S$ & Leiomyosarcoma & Rhabdomyosarcoma & $I M T$ & $S F T$ & GIST \\
\hline CD34 & + & + & - & - & - & + & + \\
\hline SMA & \pm & - & + & + & + & - & \pm \\
\hline Desmin & \pm & - & + & + & + & - & \pm \\
\hline Myogenin & - & - & - & + & - & - & - \\
\hline c-kit & - & - & - & - & \pm & - & + \\
\hline ALK-1 & - & - & - & - & + & - & - \\
\hline PR & + & + & \pm & - & - & \pm & - \\
\hline
\end{tabular}

SS, stromal sarcoma; PR, progesterone receptor; \pm , variably positive.

\section{Conclusions}

The clinical and morphologic spectrum of spindle cell lesions of the prostate demonstrates broad overlap, although the differences in patient prognosis and treatment modalities highlight the importance of rendering the correct diagnosis. In this review, we have provided an overview of the more commonly seen spindle cell lesions of the prostate, although, as a group, these are generally rare entities.

\section{References}

1 Eble JN, Sauter G, Epstein JI, et al (eds). The World Health Organization Classification of Tumors of the Urinary System and Male Genital Organs. IARC Press: Lyon, France, 2004, pp 160, 209-211.

2 Gaudin PB, Rosai J, Epstein JI. Sarcomas and related proliferative lesions of specialized prostatic stroma: a clinicopathologic study of 22 cases. Am J Surg Pathol 1998;22:148-162.

3 Bostwick DG, Hossain D, Qian J, et al. Phyllodes tumor of the prostate: long-term followup study of 23 cases. J Urol 2004;172:894-899.

4 Herawi M, Epstein JI. Specialized stromal tumors of the prostate: A clinicopathologic study of 50 cases. Am J Surg Pathol 2006;30:694-704.

5 Mobbs BG, Liu Y. Immunohistochemical localization of progesterone receptor in benign and malignant human prostate. Prostate 1990;16:245-251.

6 Srinivasan G, Campbell E, Bashirelahi N. Androgen, estrogen, and progesterone receptors in normal and aging prostates. Microsc Res Tech 1995;30:293-304.

7 Torlakovic E, Lilleby W, Berner A, et al. Differential expression of steroid receptors in prostate tissues before and after radiation therapy for prostatic adenocarcinoma. Int J Cancer 2005;117:381-386.

8 Hansel DE, Epstein JI. Sarcomatoid carcinoma of the prostate: a study of 42 cases. Am J Surg Pathol 2006;30:1316-1321.

9 Dundore PA, Cheville JC, Nascimento AG, et al. Carcinosarcoma of the prostate. Report of 21 cases. Cancer 1995;76:1035-1042.

10 Shannon RL, Ro JY, Grignon DJ, et al. Sarcomatoid carcinoma of the prostate. A clinicopathologic study of 12 patients. Cancer 1992;69:2676-2682.

11 Ray ME, Wojno KJ, Goldstein NS, et al. Clonality of sarcomatous and carcinomatous elements in sarcoma- toid carcinoma of the prostate. Urology 2006;67: 423.e5-e8.

12 Jones EC, Clement PB, Young RH. Sclerosing adenosis of the prostate gland. A clinicopathological and immunohistochemical study of 11 cases. Am J Surg Pathol 1991;15:1171-1180.

13 Sakamoto N, Tsuneyoshi M, Enjoji M. Sclerosing adenosis of the prostate: histopathological and immunohistochemical analysis. Am J Surg Pathol 1991; 15:660-667.

14 Grignon DJ, Ro JY, Srigley JR, et al. Sclerosing adenosis of the prostate. A lesion showing myoepithelial differentiation. Am J Surg Pathol 1992;16:383-391.

15 Cheville JC, Dundore PA, Nascimento AG, et al. Leiomyosarcoma of the prostate. Report of 23 cases. Cancer 1995;76:1422-1427.

16 Sexton WJ, Lance RE, Reyes AO, et al. Adult prostate sarcoma: the M.D. Anderson Cancer Center Experience. J Urol 2001;166:521-525.

17 Cheville JC, Dundore PA, Nascimento AG, et al. Leiomyosarcoma of the prostate. Report of 23 cases. Cancer 1995;76:1422-1427.

18 Kelley TW, Borden EC, Goldblum JR. Estrogen and progesterone receptor expression in uterine and extrauterine leiomyosarcomas: an immunohistochemical study. Appl Immunohistochem Mol Morphol 2004; 12:338-341.

19 Raney RB, Anderson JR, Barr FG, et al. Rhabdomyosarcoma and undifferentiated sarcoma in the first two decades of life: a selective review of intergroup rhabdomyosarcoma study group experience and rationale for Intergroup Rhabdomyosarcoma Study V. J Pediatr Hematol Oncol 2001;23:215-220.

20 Waring PM, Newland RC. Prostatic embryonal rhabdomyosarcoma in adults. A clinicopathologic review. Cancer 1992;69:755-762.

21 Nabi G, Dinda AK, Dogra PN. Primary embryonal rhabdomyosarcoma of prostate in adults: diagnosis and management. Int Urol Nephrol 2002;34:531-534.

22 Huang WL, Ro JY, Grignon DJ, et al. Postoperative spindle cell nodule of the prostate and bladder. J Urol 1990;143:824-826.

23 Cespedes RD, Lynch SC, Grider DJ. Pseudosarcomatous fibromyxoid tumor of the prostate. A case report with review of the literature. Urol Int 1996;57: 249-251.

24 Montgomery EA, Shuster DD, Burkart A, et al. Inflammatory myofibroblastic tumors of the urinary tract: A clinicopathological study of 46 cases, including a malignant example and a subset associated with high grade urothelial carcinoma. Am J Surg Pathol 2006;30:1502-1512. 
25 Perez Garcia FJ, Pinto Blazquez J, Gutierrez Garcia R, et al. Inflammatory prostatic pseudotumor (fibromyxoid pseudosarcomatous tumor). Arch Esp Urol 2004;57: 657-660.

26 Tsuzuki T, Magi-Galluzzi C, Epstein JI. ALK-1 expression in inflammatory myofibroblastic tumor of the urinary bladder. Am J Surg Pathol 2004;28: 1609-1614.

27 Takeshima Y, Yoneda K, Sanda N, et al. Solitary fibrous tumor of the prostate. Pathol Int 1997;47: 713-717.

28 Kelly PM, Baxter GM. Solitary fibrous tumour of the prostate. Br J Radiol 1998;71:1086-1088.

29 Pins MR, Campbell SC, Laskin WB, et al. Solitary fibrous tumor of the prostate a report of 2 cases and review of the literature. Arch Pathol Lab Med 2001;125:274-277.

30 Grasso M, Blanco S, Franzoso F, et al. Solitary fibrous tumor of the prostate. J Urol 2002;168:1100.

31 Sekine H, Ohya K, Kojima S, et al. Solitary fibrous tumor of the prostate. Int J Urol 2001;8:137-138.

32 Ishikawa T, Kawabata G, Terakawa T, et al. Solitary fibrous tumor in the pelvic space. Urol Res 2004;32: 49-50.

33 Mentzel T, Bainbridge TC, Katenkamp D. Solitary fibrous tumour: clinicopathological, immunohistochemical, and ultrastructural analysis of 12 cases arising in soft tissues, nasal cavity and nasopharynx, urinary bladder and prostate. Virchows Arch 1997;430: 445-453.

34 Herawi M, Epstein JI. Solitary fibrous tumor (SFT) on prostate needle biopsy or TURP, in preparation.

35 Van der Aa F, Sciot R, Blyweert W, et al. Gastrointestinal stromal tumor of the prostate. Urology 2005; $65: 388$.
36 Madden JF, Burchette JL, Raj GV, et al. Anterior rectal wall gastrointestinal stromal tumor presenting clinically as prostatic mass. Urol Oncol 2005;23:268-272.

37 Herawi M, Montgomery EA, Epstein JI. Gastrointestinal stromal tumors (GIST) on prostate needle biopsy: A clinicopathologic study of 8 cases. Am J Surg Pathol 2006;30:1389-1395.

38 Iwasaki $\mathrm{H}$, Ishiguro $\mathrm{M}$, Ohjimi $\mathrm{Y}$, et al. Synovial sarcoma of the prostate with $\mathrm{t}(\mathrm{X} ; 18)(\mathrm{p} 11.2 ; \mathrm{q} 11.2)$. Am J Surg Pathol 1999;23:220-226.

39 Pan CC, Chang YH. Primary synovial sarcoma of the prostate. Histopathology 2006;48:321-323.

40 Williams DH, Hua VN, Chowdhry AA, et al. Synovial sarcoma of the prostate. J Urol 2004;171:2376.

41 Nishiyama T, Ikarashi T, Terunuma M, et al. Osteogenic sarcoma of the prostate. Int J Urol 2001;8:199-201.

42 Bain GO, Danyluk JM, Shnitka TK, et al. Malignant fibrous histiocytoma of prostate gland. Urology 1985; 26:89-91.

43 Chin W, Fay R, Ortega P. Malignant fibrous histiocytoma of prostate. Urology 1986;27:363-365.

44 Kumala RV, Seppanen JH, Vaajalahti PJ, et al. Malignant fibrous histiocytoma of the prostate. Case report. Scand J Urol Nephrol 1994;28:429-431.

45 Chandan VS, Wolsh L. Postirradiation angiosarcoma of the prostate. Arch Pathol Lab Med 2003;127:876-878.

46 Chung AK, Michels V, Poland GA, et al. Neurofibromatosis with involvement of the prostate gland. Urology 1996;47:448-451.

47 Rames RA, Smith MT. Malignant peripheral nerve sheath tumor of the prostate: a rare manifestation of neurofibromatosis type 1. J Urol 1999;162:165-166.

48 Furihata M, Sonobe H, Iwata J, et al. Granular cell tumor expressing myogenic markers in the prostate. Pathol Int 1996;46:298-300. 